\title{
Relação entre Atenção e Desempenho em Leitura, Escrita e Aritmética em Crianças
}

\author{
Elissandra Serena de Abreu ${ }^{1}$ \\ Universidade do Vale do Rio dos Sinos, São Leopoldo-RS, Brasil \\ Vanisa Fante Viapiana \\ Pontifícia Universidade Católica do Rio Grande do Sul, Porto Alegre-RS, Brasil \\ Adriana Raquel Binsfeld Hess \\ Universidade do Vale do Rio dos Sinos, São Leopoldo-RS, Brasil \\ Hosana Alves Gonçalves, Marcia Santos Sartori \\ Pontifícia Universidade Católica do Rio Grande do Sul, Porto Alegre-RS, Brasil \\ Claudia Hofheinz Giacomoni \\ Universidade Federal do Rio Grande do Sul, Porto Alegre-RS, Brasil \\ Lilian Milnitsky Stein, Rochele Paz Fonseca \\ Pontifícia Universidade Católica do Rio Grande do Sul, Porto Alegre-RS, Brasil
}

\section{RESUMO}

Este estudo buscou verificar se há relação entre atenção (seletiva e sustentada visual) e o desempenho escolar (leitura, escrita e aritmética). Foram avaliados 258 estudantes do $1^{\circ}$ a $9^{\circ}$ ano do ensino fundamental de escolas brasileiras públicas e privadas, com idade média de 10,33 anos (DP=2,52). Os estudantes foram avaliados pelo Teste de Cancelamento do Sinos - Versão Infantil (TCS) e pelo Teste de Desempenho Escolar - Segunda Edição (TDE-II). Os escores dos testes foram correlacionados pelo coeficiente de correlação de Pearson, considerando o nível de significância de $p \leq 0,05$. Houve correlações significativas positivas de intensidade fraca entre os escores totais do TCS e TDE-II e correlação negativas significativas de intensidade fraca entre os escores de erros e omissões do TCS e escores totais do TDE-II. Foi possível observar relação entre os construtos estudados, porém sugere-se que investigações futuras utilizem diferentes métodos para avaliar outras habilidades relacionadas ao desempenho escolar e processos atencionais.

Palavras-chave: atenção; desempenho escolar; leitura; escrita; aritmética.

ABSTRACT - Relationship between Attention and Performance in Reading, Writing and Arithmetic in Children

This study sought to verify the relationship between attention (selective and sustained visual) and school performance (reading, writing and arithmetic). We evaluated 258 students from the 1st to the 9th year of elementary school in Brazilian public and private schools, with a mean age of 10.33 years $(\mathrm{SD}=2.52)$. The students were evaluated by the Bells Test - children's version $(\mathrm{BT})$ and by the School Performance Test - second edition (SPT-II). Test scores were correlated by the Pearson correlation coefficient, considering the level of significance of $p<0.05$. There were significant positive correlations of weak intensity between BT and SPT-II total scores and significant negative correlation of weak intensity between BT errors and omission scores and total SPT-II scores. An observable relationship between the constructs studied exists; however, it is suggested that future investigations use different methods to evaluate other skills related to school performance and attentional processes.

Keywords: attention; school performance; reading; writing; arithmetic.

\section{RESUMEN - Relación entre Atención y Desempeño en Lectura, Escritura y Aritmética en Niños}

Este estudio trata de determinar si existe relación entre atención (selectiva y sostenida visual) y desempeño escolar (lectura, escritura y aritmética). Fueron evaluados 258 estudiantes del 1 al 9 año de Enseñanza Primaria de escuelas públicas y privadas de Brasil, con edad media de 10,33 años ( $\mathrm{DP}=2,52)$. Los estudiantes fueron evaluados utilizando el Test de Cancelamento do Sinos-Versão Infantil (TCS) Test de Cancelamiento de las Campanas Versión Infantil y por el Test de Desempeño Escolar - Segunda Edición (TDE-II). Las puntuaciones de los tests fueron correlacionados por el coeficiente de correlación de Pearson, considerando el nivel de significación de $p<0,05$. Hubo correlaciones positivas significativas de baja intensidad entre los puntajes totales del TCS y TDE-II y correlaciones negativas significativas de baja intensidad entre los puntajes de errores y omisiones del TCS y puntajes totales de TDE-II. Fue posible observar relación entre los constructos estudiados, sin embargo se sugiere que investigaciones futuras utilizen diferentes métodos para evaluar otras habilidades relacionadas con el desempeño escolar y los procesos de atención.

Palabras clave: atención; desempeño escolar; lectura; escritura; aritmética. 
O desempenho escolar está relacionado a fatores do contexto escolar, motivacionais e cognitivos (Winne \& Nesbit, 2010). A competência em leitura, escrita e aritmética se desenvolve ao longo da progressão escolar, de acordo com a idade e o desenvolvimento neurocognitivo (Breslau et al., 2009; Capovilla \& Dias, 2008; Fonseca, Lima, Ims, Coelho, \& Ciasca, 2015). Nessa perspectiva, a Neuropsicologia cognitiva tem investigado as possíveis relações entre os mecanismos neurocognitivos, tais como a atenção, memória, funções executivas (FE) e linguagem com o desempenho escolar (DE). Dentre tais mecanismo, a atenção está entre os fatores de maior influência na aprendizagem e sucesso escolar (Posner \& Rothbart, 2014).

A atenção é um construto multidimensional e suas funções e processos são responsáveis pela seleção, controle e a ativação de informações no cérebro (Rueda, Pozuelos, \& Cómbita, 2015). Ela pode ser um processo mental involuntário, quando, por exemplo, um barulho alto desperta a atenção quer a pessoa queira ou não (Diamond, 2013); um processo controlado, quando há manutenção do foco em uma determinada tarefa por um tempo necessário para finalizá-la (atenção sustentada); e, ainda, quando há o gerenciamento de algumas informações em detrimento de outras disponíveis no meio (atenção seletiva) (Mahone \& Schneider, 2013). A atenção está, normalmente em interação com os demais mecanismos cognitivos (Hilton, Jarrett, McDonald, \& Ollendick, 2016; Oh, 2010). Dificuldades atencionais podem estar relacionadas com déficits de funcionamento da memória e também de FE (Crosbie et al., 2013; Fassbender et al., 2011; Logue \& Gould, 2014).

As formas controladas de atenção, principalmente o desempenho da atenção seletiva, estão relacionadas ao componente de controle inibitório das FE, o qual é o responsável por inibir estímulos distratores em detrimento de outros (Diamond, 2013). Por esse motivo, os processos controlados de atenção podem ser denominados como atenção executiva, tal tipo de processamento atencional é necessário em tarefas cognitivas complexas que exigem a inibição de respostas concorrentes, manutenção do objetivo e a seleção de uma resposta (Diamond, 2013; LeFevre et al., 2013).

No que tange à avaliação da atenção seletiva e sustentada, as tarefas de busca visual têm se mostrado sensíveis. Nelas o testando é convocado a encontrar figuras de um objeto específico entre outros objetos semelhantes distratores (Luck \& Mangnun, 2009). Os testes com paradigma de cancelamento têm essa característica, pois solicitam ao indivíduo a ser avaliado que faça uma varredura visual no estímulo e risque os alvos descritos previamente, entre os diversos tipos de imagens. Com esse tipo simples de tarefa é possível avaliar atenção seletiva e sustentada, velocidade de processamento e percepção visual (Cardoso, Silva, \& Fonseca, 2011; Gauthier, Dehaut,
\& Joanette, 1989; Gonçalves et al., 2013; Silva, Cardoso, \& Fonseca, 2012; Wong, 2012).

Testes de atenção seletiva visual ministrados em bebês mostram evidências do desenvolvimento desse tipo de atenção a partir dos 6 meses de vida, quando a criança consegue focar em imagens mais atraentes por um período de tempo maior, inibindo os possíveis distratores que estão a sua volta (Reynolds, Courage, \& Richards, 2013). Os processos atencionais controlados parecem assumir um importante papel no desenvolvimento cognitivo e escolar da leitura, escrita e matemática (Stevens \& Bavelier, 2012).

Nesse contexto, já está consolidado, na literatura, o papel fundamental da atenção visual no processamento da leitura tanto de crianças sem queixas quanto para aquelas com dificuldade na decodificação e/ou na compreensão leitora (Trautmann \& Zepf, 2012). Mais especificamente, o papel organizador e seletivo da atenção auxilia na análise e reconhecimento de estímulos expostos no campo visual. Acredita-se que esse mesmo mecanismo top-down ocorra durante a leitura, principalmente durante sua aquisição. Nesse caso, a criança realiza uma pesquisa em série sobre as letras da palavra apresentada para então relacioná-las entre si e, posteriormente, acessar seu significado (Vidyasagar \& Pammer, 2010).

Estudos longitudinais sugerem que o desenvolvimento da atenção visual em pré-escolares prediz a aquisição da leitura (Facoetti et al., 2006; Franceschini, Gori, Ruffino, Pedrolli, \& Facoetti, 2012), além disso, sabe-se que crianças com mal desempenho em tarefas de atenção visual no período pré-escolar tendem a apresentar dificuldades para aprender a ler (Franceschini et al., 2012). Alguns estudos defendem que uma das hipóteses para a dislexia (transtorno específico de aprendizagem da leitura) é a ineficiência ou a lentidão do deslocamento da atenção para os estímulos visuais (Hari \& Renvall, 2001; Ruffino et al., 2010; Vidyasagar \& Pammer, 2010). Nesse mesmo contexto, as dificuldades na aquisição da leitura poderiam ser explicadas pela lentidão na reorganização de busca por estímulos dispostos no campo visual, uma vez que aprender a ler exige o treinamento e a sistematização dos mecanismos de busca visual (por exemplo, realizar a busca da esquerda para a direita durante a leitura) (Vidyasagar \& Pammer, 2010).

A influência da atenção no processamento da leitura é investigada há muitos anos e já existem inclusive modelos teóricos explicando essa relação (Lobier, Dubois, \& Valdois, 2013; Wanat, 1971; Willows, 1974). Contudo, não surpreendentemente, observam-se lacunas importantes na literatura nacional e internacional a respeito do envolvimento da atenção nos processos de escrita. Encontram-se estudos defendendo o papel da habilidade de escrita na estimulação de funções cognitivas em geral, entre elas a atenção visuoespacial. Mais especificamente, diz-se que a escrita influencia diretamente a forma 
como se processam as informações espaciais, para além da linguagem (Chan \& Bergen, 2005). Os poucos estudos que discorrem sobre efeito da atenção nas habilidades de escrita reforçam o papel preditor da atenção visual principalmente nos períodos iniciais dessa aprendizagem (Plaza \& Cohen, 2007). Porém, acredita-se que a atenção seja fundamental não apenas durante a aquisição da escrita, uma vez que durante a elaboração de um texto, por exemplo, é de extrema importância que o redator controle as interferências que entram em conflito com o planejado para o alcance do objetivo (escrever um texto). Esse controle é uma das funções da atenção, mais especificamente da atenção executiva (Engle, 2002).

No que se refere à matemática, a atenção sustentada visual foi relacionada com a acuidade do número (precisão com que quantidades numéricas podem ser discriminadas), habilidade de mapear o número no espaço (por exemplo, identificar a localização de um número em uma linha não numérica) e habilidades de cálculos em estudantes de 8 a 11 anos (Anobile, Stievano, \& Burr, 2013). A atenção executiva foi relacionada tanto ao conhecimento matemático procedural quanto conceitual de crianças de $2^{\mathrm{a}}$ e $3^{\mathrm{a}}$ série (McClelland, Acock, Piccinin, Rhea, \& Stallings, 2013). Os autores desse estudo mostraram que atenção executiva prevê um crescimento na fluência aritmética das crianças de um ano para o outro. Sendo assim, a atenção é importante para a aquisição de fluência matemática. A resolução de cálculos matemáticos demanda de processos atencionais controlados principalmente nos períodos iniciais da aprendizagem matemática, de forma que a automatização dos fatos e procedimentos matemáticos diminui a necessidade de processos mentais controlados (McClelland et al., 2013; Viapiana, Giacomoni, Stein, \& Fonseca, 2016). Considera-se que a atenção auxilie na resolução das mais variadas tarefas matemáticas por possibilitar a persistência na resolução de um problema matemático até que esse seja solucionado e o foco nas informações relevantes (McClelland et al., 2013). Considera-se que, por meio da inibição de informações irrelevantes, a atenção ajuda a otimizar a performance da memória de trabalho, responsável pela manipulação dos números no momento da resolução de uma operação matemática (Stevens \& Bavelier, 2012).

Nesse sentido, alguns estudos vêm se preocupando com a relação de dificuldades atencionais com o desempenho escolar. A partir de um estudo longitudinal, Rabiner e Coie (2000), mostraram que as dificuldades atencionais nos primeiros anos de escolarização foram significativamente relacionadas com o desenvolvimento de problemas de leitura nos anos escolares posteriores. Já problemas precoces de atenção foram identificados como determinantes para o sucesso acadêmico durante todo o curso do ensino fundamental (Rabiner, Carrig, \& Dodge, 2013). Também foram verificados escores mais baixos no desempenho em matemática em crianças de 4 a 7 anos (Merrell \& Tymms, 2001) e em crianças de 7 a 11 anos com Transtorno de Déficit de Atenção e Hiperatividade (TDAH) quando comparadas com controles (Antonini et al., 2016). Ainda, ao se investigar crianças e adolescentes de 6 a 17 anos com TDAH, foi constado que os participantes obtiveram, além de pior desempenho acadêmico, menor QI e maior número de comorbidades com outros transtornos psiquiátricos do que os controles. Outro estudo verificou, em adolescentes de 11 a 19 anos com problemas comportamentais, que a falta de atenção está mais relacionada com o insucesso escolar do que hiperatividade e impulsividade (Barriga et al., 2002).

Além disso, vem se buscando estabelecer uma referência da relação da atenção com o desempenho escolar em participantes saudáveis. A exemplo, Lima, Travaini, \& Ciasca (2009) avaliaram alunos sem dificuldades escolares e os resultados mostraram que o desempenho da atenção e de $\mathrm{FE}$ foram relacionados com a aprendizagem de linguagem escrita e aritmética. Mais especificamente, identificou-se correlação negativa entre o número de erros nas tarefas e o desempenho nos testes de escrita e de aritmética. Nesse caso, quanto menor o número de erros nos testes de atenção e $\mathrm{FE}$, mais satisfatório o resultado obtido nas tarefas de avaliação do desempenho escolar. Outro estudo com crianças saudáveis, também encontrou relação entre processos de atenção, desempenho escolar e idade (Trautmann \& Zepf, 2012). Uma tendência linear indicou uma redução na velocidade cognitiva, distraibilidade e lapsos de atenção com o aumento da idade, também se obteve correlação entre o aumento de lapsos de atenção com declínio nas notas escolares.

Como se pode observar, a maioria dos estudos traz uma preocupação em buscar evidências da relação de problemas de atenção com o desempenho escolar em grupos clínicos e também como esses problemas podem afetar na aprendizagem das crianças ao longo do tempo. Porém, ainda há poucos estudos que visam a investigação de processos atencionais em crianças saudáveis e sua relação, ao mesmo tempo, com leitura, escrita e aritmética. Assim, o objetivo deste estudo é investigar a relação de diferentes dimensões da atenção (seletiva, sustentada) com o desempenho em leitura, escrita e aritmética em estudantes saudáveis do $1^{\circ}$ a $9^{\circ}$ ano no ensino fundamental.

\section{Métodos e Materiais}

\section{Amostra}

A amostra inicial deste estudo foi de 322 estudantes do $1^{\circ}$ ao $9^{\circ}$ ano do ensino fundamental de três escolas públicas e duas particulares das cidades de Porto Alegre e Novo Hamburgo, no estado do Rio Grande do Sul. Após análise dos critérios de inclusão deste estudo, quinze alunos foram excluídos por repetência, dez por histórico atual ou prévio de doenças neurológicas ou psiquiátricas, dois por suspeita de dificuldades auditivas não corrigidas, um por queixas de linguagem oral, todos avaliados pelo Questionário de Fatores de Saúde, Sociodemográficos 
e Culturais para Avaliação Neuropsicológica Infantil (Fonseca, Jacobsen, \& Pureza, 2016), respondido pelos pais e/ou responsáveis. Dezoito participantes foram excluídos por obterem nível intelectual (QI) inferior a 80 na Escala de Inteligência Wechsler Abreviada (WASI) (Yates et al., 2006). Quatorze, por apresentar sintomas de desatenção, hiperatividade e impulsividade acima do esperado avaliados por meio do Multimodal Treatment of ADHD - Swanson, Nollan na Pelhan IV (MTA-SNAPIV) (Mattos, Serra-Pinheiro, Rohde, \& Pinto, 2006) respondido pelos responsáveis e pelos professores. Foram excluídos, também, quatro estudantes por outros motivos (instabilidade emocional no momento da avaliação e alfabetização em outro idioma). Assim, a amostra final foi composta por 258 estudantes, sendo $56,2 \%$ meninas e $72,5 \%$ estudantes de escola privada, sendo $19,8 \%$ de classe A, 13,6\% de classe B1, 21,3\% de classe B2, 14\% de classe $\mathrm{C} 1,9,3 \%$ de classe $\mathrm{C} 2$ e 7\% de classe D-E, de acordo com os o Critério de Classificação Econômica Brasil (ABEP, 2016), caracterizados conforme a Tabela 1.

Tabela 1

Caracterização da Amostra

\begin{tabular}{lcc}
\hline & Média & $\begin{array}{c}\text { Desvio } \\
\text { Padrão }\end{array}$ \\
\hline Idade & 10,33 & 2,52 \\
Nível intelectual (WASI) & 103,49 & 13,95 \\
Nível socioeconômico & 34,48 & 12,94 \\
SNAP-IV (pais) & 12,13 & 9,47 \\
SNAP-IV (professores) & 5,55 & 7,61 \\
\hline
\end{tabular}

\section{Instrumentos}

Instrumentos de caracterização da amostra.

Questionário de Fatores de saúde, sociodemográficos e culturais para avaliação neuropsicológica infantil. (Fonseca et al., 2016): Esse questionário foi enviado aos pais/responsáveis para avaliar os dados pessoais, demográficos, escolares, socioeconômicos e de saúde da criança, além de hábitos culturais da família. Para compor a amostra, os estudantes não poderiam apresentar doenças neurológicas e/ou psiquiátricas, déficit auditivo e/ou visual não corrigido, bem como histórico de repetência escolar.

Critério de Classificação Econômica Brasil. (ABEP, 2016). Esse questionário foi enviado aos pais/responsáveis para avaliar o nível socioeconômico dos participantes conforme critérios de corte para a população brasileira (Classe A: 45-100 pontos; Classe: B1: 38-44; Classe B2: 29-37 pontos; Classe C1: 23-28 pontos; Classe C2: 17-22 pontos; Classes D-E: 0-16 pontos).

MTA-SNAP-IV. (Mattos et al., 2006). Questionário respondido pelos pais e professores para avaliar sintomas de desatenção e hiperatividade/impulsividade. $\mathrm{O}$ questionário apresenta 18 afirmações que correspondem aos sintomas do critério A do DSM-IV (American Psychiatric Association, 1994) para TDAH. Para compor a amostra, os estudantes não poderiam apresentar seis ou mais sintomas de desatenção e /ou hiperatividade, conforme critérios de corte do instrumento.

Escala de Inteligência Wechsler Abreviada (WASI). (Yates et al., 2006). Bateria de testes utilizada para avaliar o QI geral de pessoas entre 6 e 89 anos. Neste estudo, foram usados somente os subtestes Vocabulário, que avalia as habilidades cristalizadas ou verbais, e Raciocínio Matricial que mensura capacidade fluída ou não verbal. Para compor a amostra do estudo, os estudantes deveriam obter QI igual ou superior a 80, ponto de corte entre classificação de inteligência limítrofe e média da escala.

\section{Instrumento para a avaliação da atenção.}

Teste de Cancelamento dos Sinos - Versão Infantil (TCS). (Fonseca et al., no prelo). Essa tarefa (alfa de Cronbach $=0,81$ ) avaliou as funções de atenção seletiva e sustentada, percepção visual e velocidade de processamento. É caracterizada por ser uma tarefa lápis e papel onde o testando deve cancelar com um traço todos os sinos que possuem alça e badalo dentre distratores, como sinos sem badalo, sinos sem alça e outros objetos. Foram mensurados os Erros, Omissões, Tempo e Escore Total (acertos - erros).

\section{Instrumento para a avaliação do desempenho escolar \\ Teste de Desempenho Escolar - Segunda Edição.} (TDE-II). Como em sua versão original (Stein, 1994), esse teste tem o intuito de avaliar o desempenho escolar em leitura, escrita e aritmética. Atualmente em fase de normatização, a segunda edição pretende avaliar estudantes do $1^{\circ}$ ao $9^{\circ}$ ano (a primeira edição avalia somente até $6^{\circ}$ ano). A seguir, a descrição de cada subteste:

- Subteste Leitura do Teste de Desempenho Escolar Segunda Edição (TDE-II). (M. de L. Athayde, 2015). Esse subteste avalia a leitura de palavras isoladas. Possui duas versões: a versão A (alfa de Cronbach $=0,86$ ) possuiu 36 itens e é aplicada em estudantes do $1^{\circ}$ a $4^{\circ}$ ano; já a versão $\mathrm{B}$ (alfa de Cronbach $=0,89$ ) possui 33 itens e é aplicada em alunos do $5^{\circ}$ a $9^{\circ}$ ano. Os itens foram corrigidos de forma dicotômica (certo e errado) e a aplicação foi interrompida mediante 10 erros consecutivos.

- Subteste Escrita do Teste de Desempenho Escolar Segunda Edição (TDE-II). (M. L. Athayde, Stein, Fonseca, Mendonça Filho, \& Giacomoni, 2016). Esse subteste avalia a escrita de palavras isoladas. É composto por duas versões: A (alfa de Cronbach $=0,98$ ) e B (alfa de Cronbach $=0,86$ ), ambas com 40 palavras que diferem no nível de complexidade no momento da escrita. A versão A é aplicada nos alunos de $1^{\circ}$ a $4^{\circ}$ ano, e a versão $\mathrm{B}$, nos alunos de $5^{\circ}$ a $9^{\circ}$ ano. Os itens foram corrigidos de forma dicotômica (certo e errado) e a aplicação foi interrompida mediante 10 erros consecutivos. 
- Subteste Aritmética do Teste de Desempenho Escolar Segunda Edição (TDE-II). (Viapiana, Filho, Fonseca, Giacomoni, \& Stein, 2016). Esse subteste é composto por duas versões, de acordo com a idade do participante. A versão A (alfa de Cronbach $=0,95$ ) é composta por 37 itens que avaliam processamento numérico, capacidade de resolver e identificar as quatro operações básicas, capacidade de escrita de números decimais e noções simples de frações. Essa versão é aplicada nos alunos de $1^{\circ}$ a $5^{\circ}$ ano. Já a versão B (alfa de Cronbach $=0,97)$ é composta por 43 itens que avaliam a capacidade de resolução das quatro operações básicas em cálculos multidígitos, operações com frações, operações com números inteiros, potenciação e radiciação. Essa versão é aplicada em alunos do $6^{\circ}$ a $9^{\circ}$ ano. Os itens foram corrigidos de forma dicotômica (certo e errado) e a aplicação foi interrompida mediante seis erros consecutivos.

\section{Procedimentos}

Os dados do presente estudo fazem parte do projeto de pesquisa intitulado "Atualização do Teste de Desempenho Escolar (TDE)" que foi aprovado pelo Comitê de Ética em Pesquisa da PUCRS (parecer número 131.576 de 19/10/2012, CAAE 06624312.7.0000.5336). Mediante a assinatura do Termo de Consentimento Livre e Esclarecido, os estudantes foram autorizados pelos pais e/ou responsáveis a participar deste estudo. Estes responderam também o Questionário de Fatores de saúde, sociodemográficos e culturais para avaliação neuropsicológica infantil (Fonseca et al., 2016) e o MTA-SNAP-IV (Mattos et al., 2006) utilizados para mensurar os critérios de exclusão da amostra. O professor com maior convívio com o estudante respondeu ao MTA-SNAP-IV (Mattos et al., 2006), servindo também para avaliar os critérios de exclusão. Os instrumentos foram aplicados na escola, durante o horário de aula. Os alunos responderam a uma bateria de avaliação neuropsicológica que incluía os instrumentos usados neste estudo. A aplicação foi realizada em um encontro individual de aproximadamente 1 hora e meia de duração.

\section{Análise dos Dados}

Para verificar a relação entre a capacidade de atenção seletiva visual e o desempenho em leitura, escrita e aritmética os escores brutos de todas as tarefas, foram correlacionados entre si. Os dados foram submetidos a análises de Correlações de Pearson, considerando como fracas as correlações entre zero e 0,39 ; moderadas entre 0,40 e 0,69 e fortes entre 0,70 e 1,00 (Levin \& Fox, 2004). As análises foram feitas com a versão 17.0 do software Statistical Package for Social Sciences (SPSS).

\section{Resultados}

Os escores de Omissões, Erros, Tempo e Escore total (acertos-erros) do TCS foram correlacionados com os escores de acertos das versões A e B dos subtestes do TDE-II, conforme a Tabela 2.

Tabela 2

Dados Descritivos da Amostra em Relação com o Desempenho nos Testes

\begin{tabular}{ccccc}
\hline Instrumento & Variável & & D & DP \\
\hline \multirow{3}{*}{ TCS } & Omissões & & 5,25 & 5,36 \\
& Erros & & 0,79 & 3,36 \\
& Tempo & & 134,64 & 65,61 \\
& Escore Total & & 22,92 & 12,88 \\
\hline \multirow{2}{*}{ Leitura } & Versão A & 28,90 & 11,83 \\
& \multirow{2}{*}{ TDE-II } & Versão B & 29,69 & 3,18 \\
\cline { 2 - 5 } & \multirow{2}{*}{ Escrita } & Versão A & 24,89 & 11,79 \\
& \multirow{2}{*}{ Aritmética } & Versão B & 18,62 & 8,96 \\
& \multirow{2}{*}{} & Versão A & 19,76 & 6,67 \\
& & Versão B & 21,73 & 9,64 \\
\hline
\end{tabular}

A análise correlacional entre o TCS e o subteste de Leitura do TDE-II mostram associações significativamente positivas, de magnitude fraca, entre o Escore Total no TCS e as versões A e B do subteste de Leitura. A Versão B obteve também índices significativos de correlações negativas e de magnitude correlacional fraca com os escores de Omissões e Erros do TCS conforme Tabela 3.
As análises de correlação entre o os escores do TCS e o subteste Escrita do TDE-II mostraram a associação significativamente positiva e de magnitude fraca entre o escore total no TCS e o número de acertos na versão A do subteste de Escrita, já a Versão B não obteve índices significativos de correlação com o Escore Total do TCS. Essa versão, por sua vez, obteve correlação significativamente 
negativa e fraca com o número de Erros no TCS, conforme Tabela 4.

No que tange ao subteste de Aritmética do TDE-II, a Versão A obteve índice de correlação significativamente positivo e de magnitude fraca com o Escore Total do TCS, bem como, correlação negativa e fraca com o total de Omissões no TCS. Não houve correlações significativas entre as variáveis do TCS e a versão $\mathrm{B}$ do subteste Aritmética conforme Tabela 5.

Tabela 3

Correlações entre o Número de Acertos nas Versões A e B do Subteste de Leitura do TDE-II com os Escores do TCS

\begin{tabular}{lll}
\hline \multicolumn{1}{c}{ TCS } & $\begin{array}{c}\text { Leitura A } \\
N=121\end{array}$ & $\begin{array}{c}\text { Leitura B } \\
N=136\end{array}$ \\
\hline Omissões & $-0,13$ & $-0,26^{*}$ \\
Erros & $-0,16$ & $-0,19^{*}$ \\
Tempo & $-0,11$ & $-0,01$ \\
Escore Total & $0,22^{*}$ & $0,30^{* *}$ \\
\hline
\end{tabular}

Nota. ${ }^{* *} p \leq 0,001 ;{ }^{*} p \leq 0,05$

Tabela 4

Correlações entre o Número de Acertos nas Versões A e B do Subteste de Escrita do TDE-II com os Escores do TCS

\begin{tabular}{lcc}
\hline \multicolumn{1}{c}{ TCS } & $\begin{array}{c}\text { Escrita A } \\
N=121\end{array}$ & $\begin{array}{c}\text { Escrita B } \\
N=137\end{array}$ \\
\hline Omissões & $-0,13$ & $-0,07$ \\
Erros & $-0,14$ & $-0,18^{*}$ \\
Tempo & $-0,10$ & 0,15 \\
Escore Total & $0,20^{*}$ & 0,10 \\
\hline
\end{tabular}

Nota. ${ }^{* *} p \leq 0,001 ;{ }^{*} p \leq 0,05$

Tabela 5

Correlações entre o Número de Acertos nas Versões A e B do Subteste de Aritmética do TDE-II com os Escores do TCS

\begin{tabular}{lcc}
\hline \multicolumn{1}{c}{ TCS } & $\begin{array}{c}\text { Aritmética A } \\
N=147\end{array}$ & $\begin{array}{c}\text { Aritmética B } \\
N=109\end{array}$ \\
\hline Omissões & $-0,24^{*}$ & $-0,14$ \\
Erros & $-0,02$ & $-0,06$ \\
Tempo & $-0,05$ & 0,03 \\
Escore Total & $0,25^{*}$ & 0,16 \\
\hline
\end{tabular}

Nota. ${ }^{*} p \leq 0,05$

\section{Discussão}

Este estudo teve como objetivo investigar a relação entre atenção seletiva, sustentada e o desempenho em leitura, escrita e aritmética em estudantes saudáveis do $1^{\circ}$ a $9^{\circ}$ do ensino fundamental. Seu principal caráter inovador foi examinar a relação desses construtos com os três principais eixos da aprendizagem ao longo de todos os anos escolares do mais recente sistema de ensino fundamental brasileiro, considerando os três principais pilares da aprendizagem, inerentes a todos os demais, leitura, escrita e matemática. Os padrões correlacionais entre os Escores Totais do TCS e do TDE-II mostram a relação positiva da atenção seletiva e sustentada visual com o desempenho em leitura, escrita e aritmética, bem como, a relação negativa entre os escores de omissões e de erros do TCS.

Em relação ao desempenho dos alunos em leitura, houve correlações fracas e positivas entre o Escore Total do TCS e as Versões A e B do subteste de Leitura. Além disso, obteve-se correlações fracas e negativas entre a Versão B do subteste de Leitura e os escores de Omissões e Erros do TCS. Esses resultados sugerem que a discriminação visual e a eficiência na busca estão associadas e podem ser preditivas no desempenho da leitura (Franceschini et al., 2012; Plaza \& Cohen, 2007). Casco, Tressoldi e Dellantonio (1998), descobriram que os indivíduos com escores mais baixos em uma tarefa de cancelamento também apresentaram mais erros durante a leitura que indivíduos que tiveram escores mais altos, isso pode estar relacionado a um déficit na atenção seletiva. Além da acurácia, a capacidade atencional visual tem predito a velocidade da leitura (Lobier et al., 2013). Para se ter um domínio mais rápido e eficiente na habilidade de leitura, deve-se estar atento as características seletivas dos estímulos visuais (Plaza \& Cohen, 2007). Ressaltase que as habilidades visuais em geral podem modular o desempenho em leitura (Lobier et al., 2013).

No que se refere aos resultados desta investigação, a correlação negativa de magnitude fraca da Versão B do Subteste de Escrita com escore de Erros do TCS, sugere que desatenção e falhas no controle inibitório se associam negativamente às habilidades de escrita. Segundo Berninger, Winn, MacArthur, Graham e Fitzgerald (2006), componentes de FE que regulam a atenção, como o controle inibitório e a autorregulação, são alguns dos componentes essenciais para escrita. Para Diamond (2013), o controle inibitório é um dos principais componentes das FE e se caracteriza pela capacidade de controlar a atenção, pensamentos, comportamentos e emoções para que se chegue a um objetivo. A autorregulação seria um subcomponente do controle inibitório que envolve o controle de ações impulsivas e a resistência às tentações (Diamond, 2013). Assim, durante a escrita, o indivíduo deve focar a atenção aos objetivos da tarefa e inibir a atenção a estímulos irrelevantes (Berninger et al., 2006). Já a versão A do Subteste de Escrita mostrou correlação fraca e positiva com o Escore Total do TCS, sugerindo que maiores níveis de regulação da atenção podem auxiliar na eficiência da produção escrita, principalmente na fase de aquisição dessa habilidade (Kent, Wanzek, Petscher, Al Otaiba, \& Kim, 2014; Plaza \& Cohen, 2007; Vidyasagar \& Pammer, 2010).

No que tange ao desempenho em matemática, os resultados mostram a relação significativa apenas com escores da Versão A do Subteste de Aritmética. Esse 
resultado pode ser entendido pela relação entre atenção seletiva e executiva. Entende-se que as FE são recrutadas em tarefas novas, complexas que exigem controle mental (Diamond, 2013). Sendo assim, o desempenho de estudantes dos anos iniciais do ensino fundamental demandaria mais dos processos atencionais controlados (LeFevre et al., 2013). Nos anos finais, o desempenho em aritmética parece estar mais relacionado à memória semântica e procedural específica para matemática (Viapiana, Giacomoni, et al., 2016) que reduz a demanda executiva (Simmons, Willis, \& Adams, 2012). Em crianças de 6 e 7 anos, o desempenho em matemática é reduzido diante de interferências visuoespaciais, já em crianças com 8 e 9 anos, o desempenho é reduzido diante de interferências verbais (McKenzie, Bull, \& Gray, 2003).

Cabe ressaltar que as magnitudes correlacionais fracas são comumente encontradas nos estudos neuropsicológicos pela complexidade do funcionamento cognitivo (Reppold et al., 2015). Ao considerar a aprendizagem de leitura, escrita e aritmética, é necessário considerar o envolvimento de uma grande variedade de processos cognitivos, para além da atenção visual seletiva, sustentada. Dessa forma, considera-se que as magnitudes fracas podem ser entendidas devido à grande demanda de processos linguísticos envolvidos no desempenho escolar (Berninger, Abbott, Cook, \& Nagy, 2016). Na matemática, o número é expresso em código arábico e verbal (Dehaene \& Cohen, 1995). A Transcodificação de um código para outro é preditivo do desempenho em operações aritméticas (Moeller, Pixner, Zuber, Kaufmann, \& Nuerk, 2011). Aspectos verbais são reconhecidos no papel da consciência fonológica na predição do desempenho em leitura, escrita e aritmética (Silva, Moura, Wood, \& Haase, 2015) e, ainda, na compreensão sobre os fatos aritméticos, o significado semântico da adição e multiplicação.

Estudos defendem que a velocidade de processamento está relacionada a diversas áreas do comportamento de crianças saudáveis, mas que a associação com habilidades atencionais parece mais evidente (Jarrold, Mackett, \& Hall, 2014; Lobier et al., 2013). Nesse caso, aquelas crianças com dificuldades na velocidade de processamento podem apresentar também falhas atencionais (Jarrold et al., 2014).

O Tempo TCS obteve relações negativas com os escores do TDE-II, exceto com as Versões B de Escrita e Aritmética. A variável de tempo está relacionada à velocidade de processamento, que se refere a eficiência em realizar tarefas cognitivas básicas (Fry \& Hale, 2000). A aprendizagem em matemática envolve outros processos, como flexibilidade cognitiva e memória de trabalho, assim, buscar alvos em um estímulo fixo (como no TCS) não exige a manipulação mental de informações como quando se está resolvendo um cálculo (Rohde \& Thompson, 2007). Para esses autores, a velocidade de processamento parece ser um construto importante para a aprendizagem em matemática, porém com menos impacto se comparadas as outras. Além disso os itens da Versão B do subteste de Aritmética são mais complexos do que na versão $A$, exigindo um recrutamento maior de funções cognitivas, o que pode reduzir a velocidade de processamento, corroborando com os achados de Cowan, Shepherd, Cole-fletcher, Saxton, \& Hurry (2011) e Fuchs e colaboradores (2006). Embora as magnitudes de relação entre velocidade de processamento e desempenho escolar não sejam consensuais, em geral defende-se que ela prediz o desempenho em leitura, escrita e matemática (Christopher et al., 2012; Jones, Spurgin, Miller, \& Maricle, 2015; Lobier et al., 2013).

A medida do tempo para a execução dos subtestes do TDE-II é considerada uma limitação deste estudo. Em estudos futuros, seria interessante incluir uma tarefa de nomeação rápida para controlar os resultados ou mesmo medir o tempo de execução das tarefas do TDEII. Sugere-se, ainda, que próximos estudos investiguem a relação do desempenho escolar e a atenção a partir de uma tarefa atencional de caráter verbal, em associação às mais tradicionais não verbais, para que verifique se há um papel preditor da atenção na aquisição e desenvolvimento das habilidades escolares. Além disso, novos estudos podem também ampliar a busca de relações entre funções, subdomínios cognitivos e tipos de memória. Considerase que a avaliação das habilidades atencionais e FE, em especial da atenção seletiva e sustentada contribui no entendimento das relações entre o desenvolvimento neuropsicológico e desempenho escolar. Este estudo ressalta a importância dos processos atencionais na aprendizagem escolar e a realização de programas de intervenções, visando a melhora de habilidades atencionais antes que o aluno seja prejudicado em seu rendimento. Salienta-se, também, a importância da inclusão de avaliações neuropsicológicas em escolas e o investimento de políticas públicas, como, por exemplo, a capacitação dos professores para atentar ao desenvolvimento cognitivo de estudantes do ensino fundamental.

\section{Referências}

ABEP. (2016). Critério de Classificação Econômica Brasil. Recuperado de http://www.abep.org/criterio-brasil

American Psychiatric Association. (1994). Diagnostic and statistical manual of mental disorders (4th ed.). Washington DC: American Psychiatric Association (APA). 
Anobile, G., Stievano, P., \& Burr, D. C. (2013). Visual sustained attention and numerosity sensitivity correlate with math achievement in children. Journal of Experimental Child Psychology, 116(2), 380-391. doi: 10.1016/j.jecp.2013.06.006

Antonini, T., Kingery, K. M., Nerad, M. E., Landberg, J. M., Tamm, L., \& Epstein, J. N. (2016). Neurocognitive and Behavioral Predictors of Math Performance in Children With and Without ADHD. Journal of Attention Disorders, 20(2), 108-118. doi: 10.1177/1087054713504620

Athayde, M. de L. (2015). Desenvolvimento do Teste de Desempenho Escolar II- Subtestes de Leitura e Escrita. (Tese de Doutorado não publicada). Pontifícia Universidade Católica do Rio Grande do Sul.

Athayde, M. L., Stein, L. M., Fonseca, R. P., Mendonça Filho, E. J., \& Giacomoni, C. H. (2016). Desenvolvimento do Subteste Escrita do Teste de Desempenho Escolar II. Avaliação Psicológica, 15(2), 371-382. doi:10.15689/ap.2016.1503.10

Barriga, A. Q., Doran, J. W., Newell, S. B., Morrison, E. M., Barbetti, V., \& Dean Robbins, B. (2002). Relationships between problem behaviors and academic achievement in adolescents: The unique role of attention problems. Journal of Emotional and Behavioral Disorders, 10(4), 233-240. doi: 10.1177/10634266020100040501

Berninger, V. W., Abbott, R., Cook, C. R., \& Nagy, W. (2016). Relationships of attention and executive functions to oral language, reading, and writing skills and systems in middle childhood and early adolescence. Journal of Learning Disabilities, 50(4):434-449. doi: 10.1177/0022219415617167

Berninger, V. W., Winn, W., MacArthur, C. A., Graham, S., \& Fitzgerald, J. (2006). Implications of advancements in brain research and technology for writing development, writing instruction, and educational evolution. In Handbook of writing research (pp. 96-114).

Breslau, J., Miller, E., Breslau, N., Bohnert, K., Lucia, V., \& Schweitzer, J. (2009). The impact of early behavior disturbances on academic achievement in high school. Pediatrics, 123(6), 1472-6. doi: 10.1542/peds.2008-1406

Capovilla, A. G. S., \& Dias, N. M. (2008). Desenvolvimento de habilidades atencionais em estudantes da 1a à 4a série do ensino fundamental e relação com o rendimento escolar. Revista Psicopedagogia, 25(78), 198-211.

Cardoso, C. O., Silva, R. F. C., \& Fonseca, R. P. (2011). Teste de cancelamento dos sinos: Comparação entre duas versões. Gerais: Revista Interinstitucional de Psicologia, 4(1), 73-90.

Casco, C., Tressoldi, P. E., \& Dellantonio, A. (1998). Visual selective attention and reading efficiency are related in children. Cortex; a Journal Devoted to the Study of the Nervous System and Behavior, 34(4), 531-546. doi: 10.1016/S0010-9452(08)70512-4

Chan, T. T., \& Bergen, B. (2005). Writing direction influences spatial cognition. Proceedings of the 27 th Annual Conference of the Cognitive Science Society, (Chatterjee), 412-417. doi: 10.1038/nature09144

Christopher, M. E., Miyake, A., Keenan, J. M., Pennington, B., DeFries, J. C., Wadsworth, S. J., ... Olson, R. K. (2012). Predicting Word Reading and Comprehension with Executive Function and Speed Measures Across Development: A Latent Variable Analysis. Journal of Experimental Psychology: General, 141(3), 470-488. doi: 10.1016/j.micinf.2011.07.011.Innate

Cowan, R., Shepherd, D., Cole-fletcher, R., Saxton, M., \& Hurry, J. (2011). Basic calculation proficiency and mathematics achievement in elementary school children. Journal of Educational Psychology, 103(4), 786. doi: 10.1037/a0024556

Crosbie, J., Arnold, P., Paterson, A., Swanson, J., Dupuis, A., Li, X., ... Schachar, R. J. (2013). Response Inhibition and ADHD Traits: Correlates and Heritability in a Community Sample. Journal of Abnormal Child Psychology, 41(3), 497-507. doi: 10.1007/s10802-0129693-9

Dehaene, S., \& Cohen, L. (1995). Towards an anatomical an functional model of number processing. Math. Cogn., 1, 83-120.

Diamond, A. (2013). Executive Functions. Annual Review of Psychology, 64(1), 135-168. http://doi.org/10.1146/annurev-psych-113011-143750

Engle, R. W. (2002). Working Memory Capacity as Executive Attention. Current Directions in Psychological Science, 11(1), 19-23. doi: 10.1111/1467-8721.00160

Facoetti,A., Zorzi, M., Cestnick, L., Lorusso, M. L., Molteni, M., Paganoni, P., ... Mascetti, G. G. (2006). The relationship between visuo-spatial attention and nonword reading in developmental dyslexia. Cognitive Neuropsychology, 23(6), 841-855. doi: 10.1080/02643290500483090

Fassbender, C., Schweitzer, J. B., Cortes, C. R., Tagamets, M. A., Windsor, T. A., Reeves, G. M., \& Gullapalli, R. (2011). Working Memory in Attention Deficit/Hyperactivity Disorder is Characterized by a Lack of Specialization of Brain Function. PLoS ONE, 6(11), e27240. doi: 10.1371/journal.pone.0027240

Fonseca, G. U. S., Lima, R. F., Ims, R. E., Coelho, D. G., \& Ciasca, S. M. (2015). Evidências de validade para instrumentos de atenção e funções executivas e relação com desempenho escolar. Temas Em Psicologia, 23(4), 843-858. doi: 10.9788/TP2015.4-04

Fonseca, R. P., Jacobsen, G., \& Pureza, J. (2016). O que um bom teste neuropsicológico deve ter?. Em J. F. Sales, V. G. Haase, L. F. MalloyDiniz(Eds.), Neuropsicologia do Desenvolvimento: Infância e Adolescência. (pp. 78-91). São Paulo: Artmed.

Fonseca, R. P., Parente, M. A. M., Ortiz, K. Z., Soares, E. C. S., Scherer, L. C., Gauthier, L., \& Joanette, Y. (nd.). Teste de Cancelamento dos Sinos. São Paulo: Vetor.

Franceschini, S., Gori, S., Ruffino, M., Pedrolli, K., \& Facoetti, A. (2012). A causal link between visual spatial attention and reading acquisition. Current Biology, 22(9), 814-819. doi: 10.1016/j.cub.2012.03.013

Fry, A. F., \& Hale, S. (2000). Relationships among processing speed, working memory, and fluid intelligence in children. Biological Psychology, 54(1-3), 1-34. doi: 10.1016/S0301-0511(00)00051-X

Fuchs, L. S., Fuchs, D., Compton, D. L., Powell, S. R., Seethaler, P. M., Capizzi, A. M., ... Fletcher, J. M. (2006). The cognitive correlates of third-grade skill in årithmetic, algorithmic computation, and arithmetic word problems. Journal of Educational Psychology, 98(1), 29-43. doi: 10.1037/0022-0663.98.1.29

Gauthier, L., Dehaut, F., \& Joanette, Y. (1989). A quantitative and qualitative test for visual neglect. International Journal of Clinical Neuropsychology, 11(2), 49-54.

Gonçalves, H. A., Mohr, R. M., Moraes, A. L., Siqueira, L. de S., Prando, M. L., \& Fonseca, R. P. (2013). Componentes atencionais e de funções executivas em meninos com TDAH: Dados de uma bateria neuropsicológica flexível. Jornal Brasileiro de Psiquiatria, 62(1), 1321. doi: $10.1590 / \mathrm{S} 0047-20852013000100003$

Hari, R., \& Renvall, H. (2001). Impaired processing of rapid stimulus sequences in dyslexia. TRENDS in Cognitive Sciences, 5(12), 525-532. doi:10.1016/S1364-6613(00)01801-5

Hilton, D. C., Jarrett, M. A., McDonald, K. L., \& Ollendick, T. H. (2016). Attention Problems as a Mediator of the Relation between Executive Function and Social Problems in a Child and Adolescent Outpatient Sample. Journal of Abnormal Child Psychology, 45(4), 777-788. doi: 10.1007/s10802-016-0200-6

Jarrold, C., Mackett, N., \& Hall, D. (2014). Individual differences in processing speed mediate a relationship between working memory and children's classroom behaviour. Learning and Individual Differences, 30, 92-97. doi: 10.1016/j.lindif.2013.10.016 
Jones, A., Spurgin, A., Miller, D., \& Maricle, D. (2015). Neuropsychological domains: Executive functions: B-82 Working memory, attention, and processing speed predict academic achievement. Archives of Clinical Neuropsychology, 30(6), 553.1-553. doi: 10.1093/arclin/acv047.177

Kent, S., Wanzek, J., Petscher, Y., Al Otaiba, S., \& Kim, Y. S. (2014). Writing fluency and quality in kindergarten and first grade: The role of attention, reading, transcription, and oral language. Reading and Writing, 27(7), 1163-1188. doi: 10.1007/s11145-013-9480-1

LeFevre, J. A., Berrigan, L., Vendetti, C., Kamawar, D., Bisanz, J., Skwarchuk, S.-L., \& Smith-Chant, B. L. (2013). The role of executive attention in the acquisition of mathematical skills for children in Grades 2 through 4. Journal of Experimental Child Psychology, 114(2), 243-261. doi: 10.1016/j.jecp.2012.10.005

Levin, J., \& Fox, J. A. (2004). Estatística para ciências humanas (9th ed.). São Paulo: Pearson.

Lima, R. F. De, Travaini, P. P., \& Ciasca, S. M. (2009). Amostra de desempenho de estudantes do ensino fundamental em testes de atenção e funções executivas. Revista Psicopedagogia, 26(80), 188-199. doi: 0103-8486

Lobier, M., Dubois, M., \& Valdois, S. (2013). The role of visual processing speed in reading speed development. PLoS ONE, 8(4). doi: 10.1371/journal.pone.0058097

Logue, S. F., \& Gould, T. J. (2014). The neural and genetic basis of executive function: Attention, cognitive flexibility, and response inhibition. Pharmacology Biochemistry and Behavior, 123, 45-54. doi: 10.1016/j.pbb.2013.08.007

Luck, S. J., \& Mangnun, G. R. (2009). Attention. Em The cognitive neurosciences (4th ed., pp. 184-188). MIT Press.

Mahone, E. M., \& Schneider, H. E. (2013). Assessment of attention in preschoolers, 22(4), 361-383. doi: 10.1007/s11065-012-9217-y.Assessment

Mattos, P., Serra-Pinheiro, M., Rohde, L., \& Pinto, D. (2006). Apresentação de uma versão em português para uso no Brasil do instrumento MTA-SNAP-IV de avaliação de sintomas de transtorno do déficit de atenção/hiperatividade e sintomas de transtorno desafiador e de oposição. Revista de Psiquiatria do Rio Grande do Sul, 28(3), 290-297.

McClelland, M. M., Acock, A. C., Piccinin, A., Rhea, S. A., \& Stallings, M. C. (2013). Relations between preschool attention span-persistence and age 25 educational outcomes. Early Childhood Research Quarterly, 28(2), 314-324. doi: 10.1016/j.ecresq.2012.07.008

McKenzie, B., Bull, R., \& Gray, C. (2003). The effects of phonological and visual-spatial interference on children's arithmetical performance. Educational and Child Psychology, 20(3), 93-108.

Merrell, C., \& Tymms, P. B. (2001). Inattention, hyperactivity and impulsiveness: Their impact on academic achievement and progress. The British Journal of Educational Psychology, 71, 43-56. doi: 10.1348/000709901158389

Moeller, K., Pixner, S., Zuber, J., Kaufmann, L., \& Nuerk, H.-C. (2011). Early place-value understanding as a precursor for later arithmetic performance-A longitudinal study on numerical development. Research in Developmental Disabilities, 32(5), 1837-1851. doi: 10.1016/j. ridd.2011.03.012

Oh, S.-H. (2010). Effects of attention and working memory on perception. University of Oregon Graduate School.

Plaza, M., \& Cohen, H. (2007). The contribution of phonological awareness and visual attention in early reading and spelling. Dyslexia, 13(1), 67-76. doi: 10.1002/dys.330

Posner, M. I., \& Rothbart, M. K. (2014). Attention to learning of school subjects. Trends in Neuroscience and Education, 3(1), 14-17. doi: 10.1016/j.tine.2014.02.003

Rabiner, D. L., Carrig, M. M., \& Dodge, K. A. (2013). Attention Problems and academic achievement: Do persistent and earlier-emerging problems have more adverse long-term effects? Journal of Attention Disorders, 20(11), 946-957. doi: 10.1177/1087054713507974

Rabiner, D. L., \& Coie, J. D. (2000). Early attention problems and children's reading achievement: A longitudinal investigation. Journal of the American Academy of Child \& Adolescent Psychiatry, 39(7), 859-867. doi: 10.1097/00004583-200007000-00014

Reppold, C. T., Gomes, C. M. A., Seabra, A. G., Muniz, M., Valentini, F., \& Laros, J. A. (2015). Contribuições da psicometria para os estudos em neuropsicologia cognitiva. Revista Psicologia: Teoria e Prática, 17(2), 94-106. doi:10.15348/1980-6906/psicologia.v17n2p94-106

Reynolds, G. D., Courage, M. L., \& Richards, J. E. (2013). The Development of Attention. Oxford University Press. doi: 10.1093/ oxfordhb/9780195376746.013.0063

Rohde, T. E., \& Thompson, L. A. (2007). Predicting academic achievement with cognitive ability. Intelligence, 35(1), 83-92. doi: 10.1016/j. intell.2006.05.004

Rueda, M. R., Pozuelos, J. P., \& Cómbita, L. M. (2015). Cognitive neuroscience of attention from brain mechanisms to individual differences in efficiency. AIMS Neuroscience, 2(4), 183-202. doi: 10.3934/Neuroscience.2015.4.183

Ruffino, M., Trussardi, A. N., Gori, S., Finzi, A., Giovagnoli, S., Menghini, D., ... Facoetti, A. (2010). Attentional engagement deficits in dyslexic children. Neuropsychologia, 48(13), 3793-3801. doi: 10.1016/j.neuropsychologia.2010.09.002

Silva, J. B. L., M.oura, R. J., Wood, G., \& Haase, V. . G. (2015). Processamento fonológico e desempenho em aritmética: Uma revisão da relevância para as dificuldades de aprendizagem. Temas Em Psicologia, 23(1), 157-173. doi: 10.9788/TP2015.1-11

Silva, R. F. C. da, Cardoso, C. de O., \& Fonseca, R. P. (2012). Diferenças quanto à escolaridade em adultos no desempenho no teste de cancelamento dos sinos. Estudos de Psicologia (Natal), 17(2), 215-222. doi: 10.1590/S1413-294X2012000200004

Simmons, F. R., Willis, C., \& Adams, A.-M. (2012). Different components of working memory have different relationships with different mathematical skills. Journal of Experimental Child Psychology, 111(2), 139-155. doi: 10.1016/j.jecp.2011.08.011

Stein, L. M. (1994). TDE - Teste de desempenho escolar: Manual para aplicação e interpretação. São Paulo: Casa do Psicólogo.

Stevens, C., \& Bavelier, D. (2012). The role of selective attention on academic foundations: A cognitive neuroscience perspective. Developmental Cognitive Neuroscience, 2(1), 1-32. doi: 10.1016/j.dcn.2011.11.001.The

Trautmann, M., \& Zepf, F. D. (2012). Attentional Performance, Age and Scholastic Achievement in Healthy Children. PLoS ONE, 7(3), 1-5. doi: 10.1371/journal.pone.0032279

Viapiana, V. F., Filho, E. J. de M., Fonseca, R. P., Giacomoni, C. H., \& Stein, L. M. (2016). Development of the arithmetic subtest of the school achievement test-second edition. Psicologia: Reflexão E Crítica, 29(39), 1-10. doi: 10.1186/s41155-016-0045-5

Viapiana, V. F., Giacomoni, C. H., Stein, L. M., \& Fonseca, R. P. (2016). Evidências de validade do subteste aritmética do TDE-II : da Psicometria moderna à Neuropsicologia Cognitiva. Revista Neuropsicologia Latinoamericana, 8(2), 16-26. doi: 10.5579/rnl.2016.0306

Vidyasagar, T. R., \& Pammer, K. (2010). Dyslexia: A deficit in visuo-spatial attention, not in phonological processing. Trends in Cognitive Sciences, 14(2), 57-63. doi: 10.1016/j.tics.2009.12.003

Wanat, S. F. (1971). Linguistic structure and visual attention in reading. Cornell Univ.

Willows, D. M. (1974). Reading between the Lines: Selective Attention in Good and Poor Readers. Child Development, 45(2), 408. doi: $10.2307 / 1127962$ 
Winne, P. H., \& Nesbit, J. C. (2010). The psychology of academic achievement. Annual Review of Psychology, 61, 653-678. doi: 10.1146/ annurev.psych.093008.100348

Wong, C. E. I. (2012). Estudos neuropsicométricos com o Teste de Cancelamento dos Sinos. Pontifícia Católica do Rio Grande do Sul.

Yates, D. B., Trentini, C. M., Tosi, S. D., Corrêa, S. K., Poggere, L. C., \& Valli, F. (2006). Apresentação da Escala de Inteligência Wechsler Abreviada (WASI). Avaliação Psicológica, 5(2), 227-233.

\section{Sobre as autoras}

Elissandra Serena de Abreu é graduanda do curso de Psicologia da UNISINOS e integrante do Grupo Neuropsicologia Clínica e Experimental do Programa de Pós-Graduação em Psicologia da PUCRS.

Vanisa Fante Viapiana é Psicóloga, Especialista em Avaliação Psicológica (UFRGS). Mestre em Psicologia, área de concentração: Cognição Humana (PUCRS).

Adriana Raquel Binsfeld Hess é Psicóloga e Mestre em Psicologia Clínica pela UNISINOS e Doutora em Psicologia pela Universidade Federal do Rio Grande do Sul UFRGS. Professora do Curso de Graduação em Psicologia da UNISINOS. Colaboradora do Laboratório de Psicologia Experimental, Neurociências e Comportamento (LPNeC/UFRGS).

Hosana Alves Gonçalves é Psicóloga e Mestre em Psicologia (Cognição Humana) (PUCRS). Atualmente é doutoranda em Psicologia (PUCRS) e Professora na Faculdade de Psicologia e coordenadora do curso Neurocognição e Aprendizagem da IENH.

Márcia Santos Sartori é Fonoaudióloga e Psicopedagoga. Especialista em Linguagem com ênfase em atuação hospitalar (Centro Universitário IPA), em Saúde da Família (UFSC). Mestranda em Psicologia da Pontifícia Universidade Católica do Rio Grande do Sul (PUCRS), área de concentração: Cognição Humana (PUCRS).

Claudia Hofheinz Giacomoni é Psicóloga (PUCRS). Mestre e Doutora em Psicologia do Desenvolvimento (UFRGS). É professora do Departamento de Psicologia do Desenvolvimento e da Personalidade e do Programa de Pós-graduação em Psicologia da UFRGS e coordenadora do NEPP (Núcleo de Estudos em Psicologia Positiva).

Lilian Milnitsky Stein é Psicóloga (UFRGS). Mestre em Applied Cognitive Science pela Ontario Institute for Studies in Education e Doutora em Cognitive Psychology pela University of Arizona. Tem Pós-Doutorado pela Universitat de Barcelona . Atualmente é Professora do Programa de Pós-Graduação em Psicologia. Coordena o Grupo de Pesquisa em Processos Cognitivos (GPPC).

Rochele Paz Fonseca é Psicóloga (UFRGS) e Fonoaudióloga (ULBRA). Mestre e Doutora em Psicologia do Desenvolvimento (UFRGS), com Pós-Doutorado em Clínica e Neurociências (PUC-Rio), em Medicina (Neurorradiologia) (UFRJ) e em Ciências Biomédicas (Centro de Neuroimagem) na Universidade de Montreal. Atualmente é professora da Faculdade de Psicologia e do Programa de Pós-Graduação em Psicologia da PUCRS. Coordena o Grupo de Pesquisa Neuropsicologia Clínica e Experimental (GNCE). 